'Departamento de dermatología, Facultad de Medicina, Pontificia Universidad Católica de Chile. Santiago, Chile.

2Departamento de Dermatología, Hospital Barros Luco Trudeau, Servicio de Salud Metropolitano Sur. Santiago, Chile. ${ }^{3}$ Hospital Barros Luco Trudeau, Programa de Tuberculosis, Servicio de Salud Metropolitano Sur. Santiago, Chile.

aResidente Dermatología, Pontificia Universidad Católica de Chile, Santiago, Chile.

Trabajo no recibió financiamiento.

Los autores declaran no tener conflicto de interés.

Recibido el 8 de agosto de 2020, aceptado el 20 de abril de 2021 .

Correspondencia a: María Paz Salinas Espinoza Dirección Avenida Quilín sur 7400 casa 174 , Peñalolén. mpaz.salinas.e@gmail.com

\section{Escrofuloderma: tuberculosis cutánea y pulmonar asociada a coinfección COVID-19. Reporte de caso}

\author{
MONTSERRAT MOLGÓ ${ }^{1}$, CONSUELO CÁRDENAS $S^{1,2}$, \\ PAULINA RAMONDA ${ }^{3}$, MARÍA PAZ SALINAS ${ }^{A}$
}

\section{Scrofuloderma, cutaneous and pulmonary tuberculosis associated with COVID-19. Report of one case}

Cutaneous tuberculosis represents 1-1.5\% of extrapulmonary tuberculosis, including a variety of clinical conditions. Scrofuloderma and lupus vulgaris are the most common forms. We report a 49-year-old woman who sought medical attention through tele-dermatology concerning a cervical nodule associated with suppuration and cutaneous involvement. The diagnoses of scrofuloderma and pulmonary tuberculosis were confirmed, and during her evolution she presented a coinfection with SARS-CoV-2. The possible associations between tuberculosis and COVID-19 were reviewed.

(Rev Med Chile 2021; 149: 630-634)

Key words: COVID-19; Tuberculosis; Tuberculosis, Cutaneous; Tuberculosis, Lymph Node.
L a Tuberculosis (TB) es una enfermedad infectocontagiosa causada por el bacilo $M y$ pliamente distribuida con una tasa de incidencia global de 132 casos por 100.000 habitantes el año 2019, según estimaciones de la $\mathrm{OMS}^{1}$. Es una de las primeras 10 causas de muerte a nivel mundial, liderando la mortalidad por enfermedades infecciosas, incluso sobre el VIH/SIDA ${ }^{1}$. La incidencia en Chile durante el año 2017 fue de 15,7 casos por 100.000 habitantes, y $18,9 \%$ se manifestó con formas extrapulmonares ${ }^{2}$. De éstas, la TB cutánea representa el 1-1,5\%, incluyendo una diversidad de cuadros clínicos, siendo la escrofuloderma y el lupus vulgar las formas más comunes ${ }^{3,4}$.

Desde su primera detección en noviembre 2019 en la ciudad china de Wuhan, la pandemia generada por el Coronavirus tipo 2 del Síndrome respiratorio agudo grave, o SARS-CoV-2, ha generado gran impacto a nivel mundial, no solo en el ámbito de la salud pública, sino también por sus repercusiones económicas, políticas y sociales. La aparición de esta nueva enfermedad ha motivado el estudio de las posibles asociaciones o implicancias en otras patologías, dentro de ellas la TB.

Presentamos un caso clínico de TB con compromiso cutáneo que durante su evolución presentó coinfección con el virus SARS-CoV-2, y una revisión de la literatura. La paciente del caso clínico presentado entregó su consentimiento informado para la publicación de su historia médica y fotografías clínicas en revistas y/o congresos científicos, manteniendo la confidencialidad.

\section{Caso clínico}

Paciente de sexo femenino de 49 años de edad, de nacionalidad ecuatoriana y radicada en Chile desde hace 5 años. Sin antecedentes médicos conocidos. Inicia en enero de 2020 con aumento de volumen localizado a nivel cervical derecho, 
asintomático. Consulta en un Centro de Atención Primaria de Salud (APS), donde se le indica tratamiento con AINEs y se solicita estudio de laboratorio y ecotomografía cervical. Debido a la contingencia sanitaria nacional en contexto de pandemia COVID-19, la paciente no acude a control en APS con los resultados, y realiza una teleconsulta a dermatología en marzo de 2020. Hasta ese momento presentaba aumento progresivo del tamaño de la lesión asociado a dolor leve y un episodio de supuración mucopurulenta. Al interrogatorio no presentaba fiebre, baja de peso, compromiso del estado general, síntomas respiratorios ni otros síntomas asociados. Se observa a través de una fotografía digital un nódulo eritematoso de aproximadamente $3 \times 4 \mathrm{~cm}$ de diámetro localizado en la región cervical derecha inferior al ángulo mandibular ipsilateral, con una ulceración de aproximadamente $1 \mathrm{~cm}$ en su superficie (Figura 1). Al momento de la teleconsulta, la paciente contaba con exámenes de laboratorio recientes, destacando un hemograma sin alteraciones en las series hematológicas, 9.800 leucocitos con 60,1\% segmentados, 30,4\% linfocitos, VHS de $71 \mathrm{~mm} /$ hra, y VDRL no reactivo. Además, una ecotomografía cervical tomada durante el mes de enero informó múltiples adenopatías cervicales bilaterales, con diámetro hasta $13 \mathrm{~mm}$. Se sospecha una escrofuloderma, por lo que es derivada al Hospital Barros Luco Trudeau, donde se solicita estudio con TAC de cuello y tórax realizado a inicios de abril, que mostró un ganglio cervical derecho subcutáneo de $35 \mathrm{~mm}$ de diámetro, asociado a múltiples ganglios en cadenas cervicales derechas con aumento de tamaño y vascularización. A nivel pulmonar evidenció aislados engrosamientos pleuro intersticiales posteriores en LII, foco de mayor transparencia en segmento medial en LM, y aisladas microbulas en segmento anterior del LSD y superior del LII. Se solicita además cultivo de micobacterias en medio líquido (MGIT) de expectoración y secreción cutánea, resultando ambos positivos para Mycobacterium tuberculosis a los 5 y 13 días, respectivamente. ELISA-VIH no reactivo. La paciente inicia tratamiento para TB con esquema primario de 4 drogas a fines de marzo de 2020. A los 2 meses de tratamiento presenta cuadro respiratorio compatible con COVID-19, confirmado por PCR para SARS-CoV-2. Afortunadamente logra mantener su tratamiento antituberculoso y evoluciona de manera favorable, sin necesidad de hospitalización. A los 3 meses de tratamiento se observa buena respuesta con disminución significativa del volumen de la lesión cervical, disminución del eritema y ausencia de ulceración (Figura 2).

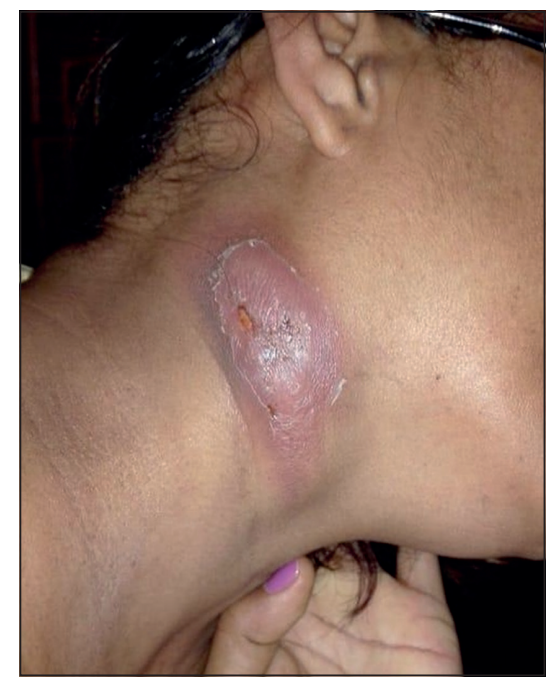

Figura 1. Nódulo eritematoso de aproximadamente $3 \times 4 \mathrm{~cm}$ de diámetro localizado en la región cervical derecha inferior al ángulo mandibular ipsilateral, con ulceración de aproximadamente $1 \mathrm{~cm}$ en su superficie.

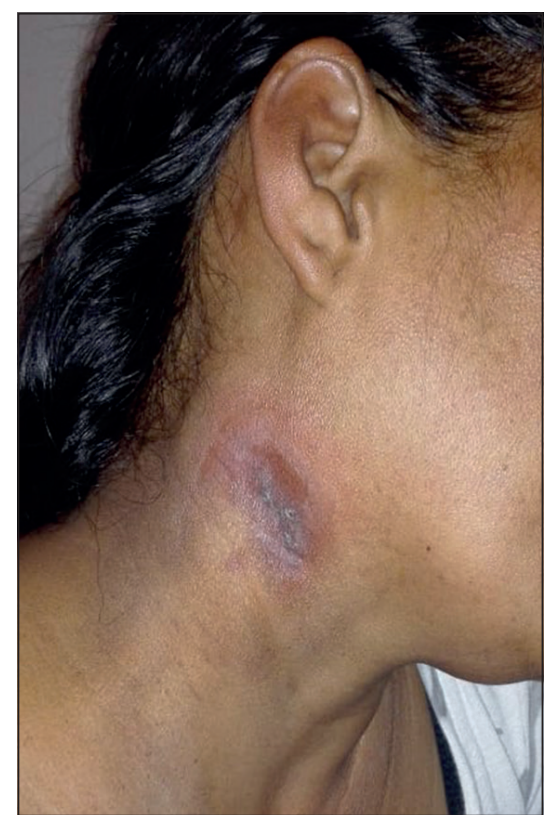

Figura 2. Tras 3 meses de tratamiento, se observa buena respuesta con disminución significativa del volumen de la lesión cervical, menor eritema y sin ulceración. 


\section{Discusión}

La escrofuloderma corresponde a una manifestación cutánea de TB que se origina a partir de su diseminación desde un foco profundo, como por ejemplo adenopatías, articulaciones, huesos, o epidídimo. Sus localizaciones más frecuentes corresponden a las áreas cervical, axilar o inguinal ${ }^{3,4}$. Clínicamente se presenta como un nódulo subcutáneo, inicialmente móvil, firme, que progresivamente aumenta de tamaño, comprometiendo la piel y pudiendo generar tractos sinuosos, ulceración y descarga de material seroso, purulento o caseoso. Estos abscesos tienen una evolución crónica, sin tratamiento tardan años en remitir dejando cicatrices hipertróficas, retracciones o secuelas atróficas ${ }^{5}$.

El cuadro clínico cutáneo puede acompañarse de síntomas generales como fiebre, baja de peso, sudoración nocturna o síntomas respiratorios, que de estar presentes, sugieren la presencia de compromiso sistémico, observándose asociación con TB pulmonar en aproximadamente $35 \%$ de los $\operatorname{casos}^{3,6}$.

El diagnóstico diferencial incluye otras patologías infecciosas como micobacteriosis atípicas, actinomicosis, micosis sistémicas o profundas como histoplasmosis o para coccidiomicosis, más frecuentes en zonas tropicales como el país de origen del caso clínico presentado, donde son consideradas endémicas. La escrofuloderma también puede simular una sífilis terciaria, enfermedad por arañazo de gato, patologías inflamatorias como acné conglobata severo o hidrosadenitis supurativa, y neoplasias como linfoma o metástasis ${ }^{3,4,6}$.

El diagnóstico requiere un alto índice de sospecha basado en la historia clínica, y su confirmación se realiza mediante estudio microbiológico, ya sea cultivo, biopsia u observación de bacilos con tinciones especiales ${ }^{5}$. La sensibilidad de los exámenes varía según el tipo de TB cutánea, siendo mayor para las formas multibacilares.

En la histopatología se observa formación de granulomas tuberculoides y necrosis caseificante, sin embargo puede ser inespecífica, mostrando un infiltrado inflamatorio crónico con escasos bacilos. Es posible utilizar tinciones como Ziehl-Neelsen para bacilos ácido alcohol resistentes en tejido o secreción, cuyo rendimiento dependerá de la carga bacteriana de la muestra. El cultivo sigue siendo el Gold Standard, pues permite diferenciar de otras micobacteriosis y evaluar el perfil de susceptibilidad antibiótica ${ }^{4,5}$, sin embargo, técnicas moleculares como la reacción de polimerasa en cadena han mostrado sensibilidad y especificidad globales de 88 y $99 \%$ respectivamente para muestras pulmonares, y sensibilidad de $85 \%$ en tejido o aspirado de ganglio ${ }^{7}$, resultando útiles al permitir diferenciar de micobacteriosis atípicas, principalmente en pacientes inmunocomprometidos, considerando que los cultivos líquidos (MGIT) pueden tardar hasta 45 días en resultar positivos ${ }^{5,6}$.

El tratamiento de la escrofuloderma y otras formas de TB cutánea es el mismo que para TB pulmonar, de acuerdo a las normas nacionales para el control y la eliminación de la tuberculosis en Chile.

En el presente caso clínico, la paciente cursó una coinfección por COVID-19 durante los primeros meses de tratamiento antituberculoso, por lo que presentamos una breve revisión de la literatura sobre esta asociación. Hasta el 20 de diciembre de 2020, el SARS-CoV-2 ha infectado cerca de 75,1 millones de personas, ocasionando más de 1,68 millones de muertes a nivel mundial ${ }^{9}$. Liu y cols. reportaron que la presencia de TB latente o activa en pacientes hospitalizados por COVID-19 actuó como factor de susceptibilidad para desarrollar enfermedad sintomática y de mayor gravedad ${ }^{10}$. La primera cohorte publicada que incluyó 49 pacientes con TB antigua o en tratamiento que desarrollaron infección por SARS-CoV-2, describió que cerca del 90\% fueron sintomáticos y $12 \%$ murió por neumonía COVID-19, ambas cifras mayores que la población general ${ }^{11}$. En una cohorte sudafricana con más de 3 millones de pacientes, con o sin COVID-19, aquellos con historia previa o actual de TB, y TB asociada a VIH tuvieron mayor riesgo de muerte por COVID-19 ${ }^{13}$. Pese a sus limitaciones, estos trabajos sugieren un perfil de mayor riesgo en pacientes con TB previa o concomitante.

Expertos en inmunología y microbiología han concluido una probable asociación entre TB y peor pronóstico de COVID-19 ${ }^{14,15}$. Si bien no hay estudios experimentales, el conocimiento actual sugiere que podrían actuar de forma sinérgica dado que ambas generan una respuesta proinflamatoria en el parénquima pulmonar, pudiendo conducir a una respuesta exacerbada, tormenta de citoquinas y daño tisular, siendo TNF e IFNlas citoquinas principales ${ }^{15}$. IL-4 e IL-13 también 
se han asociado con daño y peor pronóstico para TB y COVID-1915,17,18. Se ha descrito linfopenia en casos de coinfección, que alteraría la respuesta inflamatoria ${ }^{16}$. Esta desregulación inmunitaria se asocia además a aumento de neutrófilos a nivel pulmonar ${ }^{16,17}$. La relación linfocitos/neutrófilos podría constituir un marcador de riesgo para ambas patologías ${ }^{15,19,20}$. Finalmente, los cambios macroestructurales secundarios a TB comprometen el funcionamiento y defensa del parénquima, generando mayor susceptibilidad a complicaciones graves. Esto podría explicar el aparente peor pronóstico de pacientes con antecedente previo de TB frente a COVID-1912,13,14. Es esperable que en los próximos meses surja nueva evidencia que permita confirmar o descartar estas hipótesis ${ }^{15}$.

En conclusión, debe sospecharse coinfección con TB en pacientes cursando COVID-19 con clínica respiratoria o imágenes de tórax atípicas, aquellos con un curso de enfermedad tórpido o prolongado ${ }^{12}$. Resulta fundamental facilitar $y$ asegurar que los pacientes con TB mantengan su tratamiento observado y supervisado durante este período de pandemia y confinamiento, para prevenir complicaciones asociadas al abandono de terapia.

\section{Referencias}

1. World Health Organization. Global tuberculosis report 2019. Geneva, Switzerland: World Health Organization; 2019. Disponible en: https://www.who.int/tb/publications/global_report/en/[Consultado el 15 de julio de 2020].

2. Escobar N. Situación epidemiológica de la tuberculosis en Chile en el escenario global 2018. Rev. chil. enferm. respir. 2019; 35 (1): 63-70.

3. L van Zyl, J du Plessis, J Viljoen. Cutaneous tuberculosis overview and current treatment regimens. Tuberculosis 2018; 95: 629-38.

4. Franco-Paredes C, Marcos LA, Henao-Martínez AF, et al. Cutaneous mycobacterial infections. Clin Microbiol Rev. 2018; 32 (1): e00069-e18.

5. Khadka P, Koirala S, Thapaliya J. Cutaneous Tuberculosis: Clinicopathologic Arrays and Diagnostic Challenges. Dermatology Research and Practice 2018; 1-9.

6. Concha M, Fich F, Rabagliati R, Pinto C, Rubio R, Navea $\mathrm{O}$, et al.Tuberculosis cutánea: reporte de dos casos y revisión de la literatura. Rev Chilena Infectol 2011; 28 (3): 262-8.

7. MINSAL. MANUAL OPERATIVO Implementación del
GeneXpert MTB/ RIF en el Programa de Tuberculosis. Programa de Control y Eliminación de la Tuberculosis. Ministerio de Salud, Santiago de Chile - 2014. 2017.

8. MINSAL. Normas técnicas para el control y la Eliminación de la tuberculosis. Subsecretaría de salud pública. División de prevención y control de enfermedades. Programa nacional para el control y la eliminación de la tuberculosis. Ministerio de Salud, Santiago de Chile 2014.

9. WHO Coronavirus Disease (COVID-19) Dashboard. Disponibe en: https://covid19.who.int/ [Consultado el 30 de julio de 2020].

10. Liu Y, Bi L, Chen Y, Wang Y, Fleming J, Yu Y. Active or latent tuberculosis increases susceptibility to COVID-19 and disease severity [Internet] Infectious Diseases (except HIV/AIDS). Mar 2020. Disponible en: https://www. medrxiv.org/content/10.1101/2020.03.10.20033795v1 [Consultado el 15 de julio 2020].

11. Tadolini M, Codecasa LR, García-García JM, Blanc F, Borisov S, Alffenaar J. Active tuberculosis, sequelae and COVID-19 co-infection: first cohort of 49 cases. Eur Respir J. 2020; 56: 2001398.

12. Kumar R, Bhattacharya B, Meena V, Soneja M, Wig N. COVID-19 and TB co-infection - 'Finishing touch" in perfect recipe to 'severity' or 'death'. J Infect. 2020 July 4; 11: 39.

13. Davies M-A. HIV and risk of COVID-19 death: a population cohort study from the western cape province, South Africa. Author: western cape department of health in collaboration with the national institute for communicable diseases, South Africa. Corresponding aut. MedRxiv. $2020 ; 1-21$.

14. Min Ong CW, Migliori GB, Raviglione M, MacGregor-Skinner G, Sotgiu G, Alffenaar J-W, et al. Epidemic and pandemic viral infections: impact on tuberculosis and the lung. A consensus by the world association for infectious diseases and immunological disorders (WAidid), global tuberculosis network (GTN) and members \# of ESCMID study group for myco. Eur Respir J. 2020: 2001727.

15. Mousquer GT, Peres A, Fiegenbaum M. Pathology of TB/COVID-19 Co-Infection: The phantom menace. Tuberculosis (Edinb) 2020 Nov 17; 126: 102020.

16. Wu Y, Chen Y. Reduction and functional exhaustion of $\mathrm{T}$ cells in patients with coronavirus disease 2019. Front Immunol. 2020; 11: 1-7.

17. Busatta C, Paula V De, Luise M, Azevedo V, Paula A, Martins C. IL-4/IL-13 remodeling pathway of covid-19 lung injury. Res Sq. 2020: 1-14. https://doi. org/10.21203/rs.3.rs-34688/v1.

18. Heitmann L, Dar MA, Schreiber T, Erdmann H, Beh- 
rends J, Mckenzie ANJ, et al. The IL-13/IL-4R? axis is involved in tuberculosis-associated pathology. J Pathol. 2014; 338-50. https://doi.org/10.1002/path.4399.

19. Muefong CN, Sutherland JS. Neutrophils in tuberculosis- associated inflammation and lung pathology. Front Immunol. 2020; 11: 1-9. doi: 10.3389/fim- mu.2020.00962.

20. Liu Y, Du X, Chen J, Jin Y, Peng L, Wang HHX, et al. Neutrophil-to-lymphocyte ratio as an independent risk factor for mortality in hospitalized patients with COVID-19. J Infect. 2020; 81: 6-12. https://doi. org/10.1016/j.jinf.2020.04.002. 